\title{
Regional Grey Matter Microstructural Changes and Volume Loss Regarding Disease Duration in Multiple Sclerosis Patients
}

Elisabeth Solana ( $\square$ elisabeth.solana@idibaps.org)

Consorci Institut D'Investigacions Biomediques August Pi I Sunyer

\section{Eloy Martinez-Heras}

Consorci Institut D'Investigacions Biomediques August Pi I Sunyer

Victor Montal

Biomedical Research Institute Sant Pau

\section{Eduard Vilaplana}

Biomedical Research Institute Sant Pau

Elisabet Lopez-Soley

Consorci Institut D'Investigacions Biomediques August Pi I Sunyer Joaquim Radua

Consorci Institut D'Investigacions Biomediques August Pi I Sunyer

Nuria Sola-Valls

Consorci Institut D'Investigacions Biomediques August Pi I Sunyer

Carmen Montejo

Consorci Institut D'Investigacions Biomediques August Pi I Sunyer

Yolanda Blanco

Consorci Institut D'Investigacions Biomediques August Pi I Sunyer Irene Pulido-Valdeolivas

Consorci Institut D'Investigacions Biomediques August Pi I Sunyer

Maria Sepúlveda

Consorci Institut D'Investigacions Biomediques August Pi I Sunyer

\section{Magi Andorra}

Consorci Institut D'Investigacions Biomediques August Pi I Sunyer

\section{Joan Berenguer}

Hospital Clinic of Barcelona

\section{Pablo Villoslada}

Consorci Institut D'Investigacions Biomediques August Pi I Sunyer Martinez-Lapiscina EH

Consorci Institut D'Investigacions Biomediques August Pi I Sunyer

\section{Ferran Prados}

Universitat Oberta de Catalunya

Albert Saiz 
Consorci Institut D'Investigacions Biomediques August Pi I Sunyer

Juan Fortea

Biomedical Research Institute Sant Pau

Sara Llufriu

Consorci Institut D'Investigacions Biomediques August Pi I Sunyer

\section{Research Article}

Keywords: grey matter, DTI, volume, disease course, multiple sclerosis

Posted Date: April 13th, 2021

DOI: https://doi.org/10.21203/rs.3.rs-403709/v1

License: (c) (P) This work is licensed under a Creative Commons Attribution 4.0 International License. Read Full License

Version of Record: A version of this preprint was published at Scientific Reports on August 19th, 2021. See the published version at https://doi.org/10.1038/s41598-021-96132-x. 


\section{Abstract}

The spatio-temporal characteristics of grey matter (GM) impairment in multiple sclerosis (MS) are poorly understood. We used a surface-based diffusion MRI processing tool to investigate regional modifications of microstructure and volume loss in GM at different time-points of the disease, and their relationship to disability. We studied 54 healthy controls and $247 \mathrm{MS}$ patients classified according to disease duration: MS1 (less than 5 years, $n=67)$; MS2 (5-15 years, $n=107)$; and MS3 (more than 15 years, $n=73$ ). We compared GM mean diffusivity (MD) and volume between groups, and estimated their clinical correlations. Regional modifications in MD and volume did not overlap early in the disease, and became widespread in later phases. We found higher MD in MS1 group, mainly in the temporal cortex, and volume reduction in right putamen. Additional MD changes were evident in cingulate and occipital cortices in the MS2 group, coupled to volume reductions in deep GM and parietal and frontal poles. Changes extended to more than $80 \%$ of regions in MS3 group. Clinical disability was correlated with GM changes. Microstructural integrity loss and atrophy present differential spatial predominance early in MS and accrual over time, probably due to distinct pathogenic mechanisms that underlie tissue damage.

\section{Introduction}

Multiple sclerosis (MS) is a chronic inflammatory, demyelinating and neurodegenerative disease of the central nervous system that leads to physical and cognitive disability ${ }^{1}$. Early studies considered white matter (WM) damage as a hallmark of MS, although advances in immunopathology and magnetic resonance imaging (MRI) techniques have highlighted the important role of grey matter (GM) damage in the pathogenesis of this disease ${ }^{2,3}$. Perivenous and confluent demyelinated lesions, as well as subpial cortical demyelination are evident in the GM of MS patients, with diffuse changes only partially related to focal injury ${ }^{4}$. Indeed, damage to the cortical and deep GM seems to be closely linked to the progression of physical and cognitive disability $3,5,6$.

Alterations of the GM in MS have been investigated in vivo through different approaches ${ }^{7-9}$. In the context of MS, diffusion tensor imaging (DTI) has the potential to provide quantitative measurements of microstructural changes in GM. However, inconsistencies in the presence and direction (increases or decreases) of the mean diffusivity (MD) and in fractional anisotropy $(F A)^{6,9}$, highlight the technical challenge present when studying diffusion metrics in the cortex. Volumetric studies have also identified patterns of GM atrophy across the brain that seem to predominate in eloquent areas in the early stages of the disease, such as in the thalamus, posterior cingulate cortex and precuneus ${ }^{7}$, and volume loss seems to evolve at different rates over the course of the disease ${ }^{10,11}$. However, it is not fully clear whether the rates of these microstructural and volumetric changes in GM emerge in parallel or if they follow different trajectories.

It is possible that a new surface-based diffusion methodology may improve the assessment of changes in integrity to the cortical microstructure of the GM in MS. This approach, which reduces the partial volume effects $^{12-14}$, has been applied to other neurodegenerative diseases like Alzheimer's disease and frontotemporal dementia where it offers superior sensitivity in mapping cortical GM changes. By adopting this approach, the present study aimed to characterise the regional GM damage, both in cortical and deep GM, analysing the spatial distribution and overlap of diffusion metrics and volume changes in MS patients at three different time- 
points of the disease course. Moreover, these modifications were mapped to disease activity and clinical disability.

\section{Methods}

\section{Participants}

For this cross-sectional study, we included a cohort of 271 patients with relapsing-remitting or secondary progressive MS according to the 2010 McDonald criteria ${ }^{24}$ prospectively recruited at the MS Unit in the Hospital Clinic of Barcelona, as well as 54 healthy controls (HC) without any prior or present history of neurological or psychiatric conditions. To explore GM changes at different time-points of the disease, patients were classified into three groups according to their disease duration: less than 5 years from disease onset (MS1); from 5 to 15 years (MS2); and more than 15 years of disease duration (MS3) ${ }^{25}$.

Physical disability was evaluated in all patients using the Expanded Disability Status Scale (EDSS) ${ }^{24}$, and in 214 of them, attention and information processing speed was assessed using the Symbol Digit Modalities Test and Paced Auditory Serial Addition Test from the Brief Repeatable Battery of Neuropsychological tests ${ }^{26}$. We calculated a mean z-score of these two tests (zAttention-Processing speed) using the age-and educationadjusted values of a healthy Spanish cohort ${ }^{27}$.

The Ethics Committee of the Hospital Clinic of Barcelona approved the study and all the participants signed an informed consent before enrolment onto the study. All procedures were performed according to the principles of the Helsinki Declaration.

\section{MRI acquisition and processing}

\section{Structural and diffusion MRI acquisition}

MRI was performed on a 3T Magnetom Trio (SIEMENS, Erlangen, Germany) scanner with a 32 channel phasedarray head coil. The protocol included structural 3D-Magnetization Prepared Rapid Acquisition Gradient Echo (MPRAGE), 3D-T2 fluid-attenuated inversion recovery (FLAIR) and diffusion-weighted imaging (DWI) sequences. DWI was acquired with two different sequence parameters. A detailed description of all sequences used in this study is available in the Supplementary material ${ }^{10,28}$.

\section{Grey matter volume processing}

Before computing GM volume, WM lesions were segmented in 3D-MPRAGE space with ITK-SNAP Software (http://www.itksnap.org/pmwiki/pmwiki.php). We used a linear registration of the 3D-FLAIR image to align with 3D-MPRAGE to improve both the identification and delineation of lesions. Subsequently, we applied a WM lesion-filling approach ${ }^{29}$ and obtained $76 \mathrm{GM}$ regions using the Mindboggle software (https://mindboggle.info), applying the Desikan-Killiany-Tourville cortical labelling atlas (31 cortical labels per hemisphere) ${ }^{30}$, and the automated subcortical segmentation offered by the FSL-FIRST package for seven subcortical regions in both hemispheres ${ }^{31}$. The resulting cortical surface parcellation was visually checked and fixed to guarantee the quality of the cortical segmentation labels for further statistical analysis. We excluded 24 patients due to segmentation errors in the cortical surface reconstruction. Finally, we normalised the 76 GM volumes using a 
volumetric scaling factor provided by SIENAX to reduce the effect of the variability in head-size for quantification ${ }^{32}$. The flowchart for GM volumetric processing is summarised in Figure 1 (left panel).

\section{Grey matter diffusion processing}

The diffusion MRI pre-processing pipeline was described in detail elsewhere ${ }^{23,28}$ and it involved the following steps: DWI denoising, Gibbs ringing correction, motion-induced distortion correction, a phase unwrapping procedure to correct for geometric distortion and bias field correction ${ }^{33}$. Subsequently, the MD DTI index was obtained $^{34}$.

The GM cortical reconstruction process was performed using in-house surface-based approach developed to measure the microstructural changes in neurodegenerative disorders ${ }^{12,13,35}$. We used Freesurfer tools to calculate the midpoint of each individual's cortical surface in order to avoid contamination by adjacent WM and cerebrospinal fluid ${ }^{12,13}$. We then registered the $T 1$-weighted image to the pre-processed diffusion data using a boundary-driven algorithm to realign both the cortical surface mask generated and the $76 \mathrm{GM}$ labels on the DTI maps of each subject ${ }^{36}$. We selected only those voxels labelled as the midpoint cortical mask to obtain the average of the diffusion metrics for each label. We then eroded each of the 14 deep GM structures using a 3 $\mathrm{mm}$ box kernel to minimise the partial volume effects and computed the MD average within these masks. The GM diffusion processing pipeline is summarised in Figure 1 (right panel).

Diffusion and volumetric data were harmonised using the ComBat function in R software ${ }^{37,38}$ to reduce the variability between sequences.

\section{Statistical analysis}

We described the demographic, clinical and neuroimaging data with the mean and standard deviation (SD, for continuous data), or as absolute numbers and proportions (for categorical data), assessing the former's normal distribution with histograms and Shapiro-Wilk tests. We analysed the demographic and clinical differences among groups with a Chi-squared or Kruskal-Wallis $\mathrm{H}$ test, using Dunn's test when necessary, and we compared the GM diffusion and volumetric data with one-way ANOVA, using Tukey HSD test for the two-group differences (HC vs. MS1, MS2 and MS3). We described the effect size of these differences using Hedges' $\mathrm{g}$. To ensure that age differences between the $\mathrm{HC}$ and MS3 group did not confound the results, we additionally calculated the changes in the MRI metrics between these two groups with a Student t-test or Mann Whitney U Test selecting only the older $\mathrm{HC}$ subjects. Finally, in the whole sample of MS patients, we calculated Spearman's correlations between the GM diffusion or volume and measures of disease severity (number of relapses, normalised lesion volume and EDSS), and the mean z-Attention-Processing speed score.

In all the analyses, we included age as a covariate to control for its potential influence on the results. We considered diffusion and volumetric values less than or greater than the $5^{\text {th }}$ and $95^{\text {th }}$ percentile as outliers, and removed them from the analysis. We used the false discovery rate (FDR) to correct for multiple comparisons, and we set the significance level at a corrected $p<0.05$. The R Statistical Software (version 3.6.1, www.Rproject.org) was used for all statistical analyses.

\section{Data availability}


The datasets generated during and/or analysed during the current study are available from the corresponding author on reasonable request.

\section{Results}

In this study, 247 MS patients were enrolled (172 women), with a mean age of $42.5 \pm 10$ years, with $11.6 \pm 9.1$ years of disease duration and with a median EDSS of 2.0 (range 0 - 7.0), as well as 54 HC. Demographic and clinical data of the final cohort are summarised in Table 1. The MS patients were divided into three groups according to disease duration, with 67 patients classified as MS1, 107 as MS2 and 73 patients as MS3. As expected, the MS3 patients were older than those in the other groups, they suffered greater disability and there was a higher proportion of secondary progressive patients in this group.

\section{Regional distribution of GM diffusion and volume changes relative to disease duration.}

When compared to $\mathrm{HC}$, there were changes in the MD of the MS1 group over five GM regions (6.6\%), mainly involving the temporal lobe, while GM volume was reduced in the right putamen. Both MD and GM changes had moderate effect size (corrected $p<0.05$ : Figure 2).

In the MS2 group, MD increased in 18 regions (23.7\%) relative to the $\mathrm{HC}$, in which most of the nodes showed a medium effect size. The largest differences between these groups were evident in areas like the bilateral cingulate, left insula and left temporal cortex (corrected $\mathrm{p}<0.05$ ). In addition, in this group of patients, there was a volume decrease in 16 regions (21.1\%) mainly involving bilateral regions of the deep GM and left parietal cortex (corrected $\mathrm{p}<0.05$ : Figure 3).

And finally, in the MS3 group, there were 61 regions (80.3\%) that showed widespread medium-to-large increase in $\mathrm{MD}$ relative to the $\mathrm{HC}$, mainly in the cerebral cortex (corrected $\mathrm{p}<0.05$ ), while $\mathrm{MD}$ in the right pallidum was dampened (corrected $p=0.001$ ). Additionally, patients of the MS3 group showed a diffuse reduction in GM volume in 68 regions (89.5\%). Bilateral areas involving the insula, frontal and parietal, and right occipital regions displayed the strongest differences between groups (corrected $p<0.05$ : Figure 4). Exploring the differences between the older subgroup of $\mathrm{HC}(47.1 \pm 5.7$ years, $n=28$ subjects) and MS3 (50.2 \pm 8.0 years, $p=0.063)$, we found that patients with MS had a higher MD in 60 nodes (78.9\%) and small diffuse volume in 62 nodes $(81.6 \%)$ throughout the cerebral cortex (corrected $\mathrm{p}<0.05)$, similar to the results with the whole HC cohort.

\section{Overlap between diffusion and volume changes relative to disease duration}

There was no overlap in regions with increased MD and volume reduction in patients with MS in the first five years of disease duration (Figure 2, bottom images), and only the left transverse temporal and right precuneus showed both GM changes in MS2 patients (Figure 3, bottom images). On the contrary, 55 nodes (72.4\%) showed both increased MD and reduced volume in patients of the MS3 group (Figure 4, bottom images).

\section{Association between modifications to GM diffusion metrics and in volume with the clinical characteristics}

The increase in MD evident in MS patients was widely associated with higher WM lesion volume (78.9\%, median rho $=0.345)$ and a worse EDSS score $(78.9 \%$, median rho $=0.233)$. The bilateral lingual, supramarginal, and isthmus of the cingulum, the superior temporal from the left hemisphere and the right postcentral cortex 
were the areas with the strongest associations. In addition, the MD changes in patients were correlated with a worse zAttention-Processing speed in $5.3 \%$ of the nodes (median rho $=-0.217$ ), particularly those involving temporal areas.

The GM volume reductions were broadly correlated with higher lesion volume $(76.3 \%$, median rho $=-0.311)$, more relapses $(60.6 \%$, median rho $=-0.179)$ and a higher EDSS score $(64.5 \%$, median rho $=-0.242)$ in MS patients. Areas of the deep $\mathrm{GM}$, and the frontal and superior parietal regions were those with the strongest associations. Finally, the GM volume changes were correlated with lower scores of zAttention-Processing speed in $75 \%$ of the regions (median rho $=0.184$ ), mainly in the bilateral posterior cingulate and thalamus, left superior temporal and putamen, and right superior parietal cortex.

\section{Discussion}

By applying a new diffusion MRI processing pipeline to GM regions in combination with volumetric measurements, this study reports anatomical changes to cortical and deep brain structures that are related to disease duration in patients with MS. We found regional modifications of microstructural tissue architecture and tissue loss within the first five years of the illness. Early in the disease, changes to microstructural barriers predominated in the cortex, while volume loss was mainly linked to deep brain structures, without overlap between these changes. GM modifications became more evident and widespread with longer disease duration. Thus, more than fifteen years after disease onset, almost all the cortex and the deep GM displayed extensive damage, which was associated with physical and cognitive disability.

Our results indicate that MD is sensitive to GM damage in patients with MS already at the beginning of the illness. The higher MD values in patients during the first five years of the disease were mainly limited to the temporal and cingulate cortices. With longer disease duration, MD also increased in other areas of the parietal and occipital cortex, and later on, his rise was seen in almost the entire brain. The early diffusion damage to the cerebral cortex supports the susceptibility of these areas to the loss of integrity of microstructural barriers ${ }^{15}$ facilitating free diffusivity ${ }^{16}$. Post-mortem studies have related MD values in the WM with myelin content, and with axonal count to a lesser extent ${ }^{17}$. The modifications observed here may be linked to the early cortical demyelination, at least in part, driven by meningeal inflammation ${ }^{18}$.

Brain atrophy is one of the main features of MS disease progression and it is thought to be uneven across the brain, indicating that some regions are more vulnerable to damage than others $7,8,10$. We found that the right putamen was the first region to lose volume, after which, atrophy accrued in the bilateral thalamus, nucleus accumbens, inferior parietal, precuneus and frontal pole. After considerable years of disease duration, almost the entire brain showed GM atrophy in patients with MS and similar patterns have been observed previously, reflecting the susceptibility of deep GM nuclei at early stages of the disease ${ }^{19,20}$. The vulnerability of deep areas to neuro-axonal loss and neuronal shrinkage could be explained by their widespread connectivity with other brain areas, occurring both through marked Wallerian degeneration ${ }^{21}$, and the presence of both diffuse oxidative injury and neurodegenerative mechanisms such as iron accumulation ${ }^{19,20}$.

One relevant finding from the analysis carried out here is the lack of overlap between the areas with changes in diffusion MRI and volume in the first years of the disease course, with a cortical preference for MD changes and 
the deep GM for volume modifications. Moreover, MD effect sizes were stronger than those of volume loss at the beginning, suggesting that MD is initially more sensitive to damage. In the later phases of MS, cortical volume reductions also emerged. Both DTI and volume modifications were associated with the WM lesion load. These results and the lack of regional co-localization endorses the hypothesis that neurodegeneration in the cortex is partially independent of cortical demyelination ${ }^{22}$.

Damage in cortical and deep GM has been associated with the progression of clinical and cognitive disability 3,5,6,11. Indeed, in this study, both increased MD and reduced GM volume were correlated with worse physical and cognitive performance. In general, changes to deep GM nuclei, and to the frontal and parietal lobes were the regions most strongly associated with clinical characteristics. GM volume reduction, probably reflecting persistent and final damage, was more closely associated with cognitive performance than diffusion metrics. In particular, reduced GM volume in the insula, parietal and deep GM was related to worse attention, working memory and psychomotor speed in patients with MS. These multimodal areas and their connections are thought to be relevant to maintain superior functions ${ }^{23}$.

This study has several strengths. First, we evaluated cortical damage using an improved technique to compute diffusion metrics that is very sensitive to describe cortical changes in other neurological diseases. Second, we combined two approaches to characterise the damage to the GM, complementary methods that provide information on different pathological changes in these areas. The results support the use of these techniques in clinical trials to assess neuroprotective therapies. Our study also has some limitations. We set out to understand the GM involvement in MS in terms of disease duration but we did not obtain differential information on lesions and normal-appearing GM. Diffusion modifications were only assessed with MD due to the isotropic characteristics of GM that difficults the use of other metrics such as FA. Although the patients with a longer disease duration were older than the $\mathrm{HC}$, the results remained unchanged when we balanced the cohorts for age by comparing MRI metrics with the older $\mathrm{HC}$ individuals. Attention and processing speed were assessed with the SDMT and PASAT, thus the use of a full neuropsychological battery will be necessary to support our findings on other cognitive domains. Finally, we followed a cross-sectional approach that allows patients with a wide range of disease duration to be compared, although further studies using longitudinal data will be needed to shed light on brain MS damage dynamics in a prospective manner.

To conclude, patients with MS suffer local modifications to their tissue microstructure and a loss of GM volume early in the disease course, which become widespread at later stages and that are related to disability. Initially, changes to diffusion metrics predominate in the cortex while atrophy is restricted to deep GM regions, with weak spatial co-localization. Such results provide relevant information on the spatial modifications of tissue changes and support the presence of several pathogenic mechanisms underlying the evolution of damage over time, accounting for GM impairment in MS, although longitudinal studies are needed to further understand the dynamics of those changes.

\section{Declarations}

\section{Acknowledgements}

The authors are grateful to Dr Núria Bargalló, Cesar Garrido and the IDIBAPS Magnetic resonance imaging facilities for their support during the realization of the study (project IBPS15-EE-3688 cofunded by MCIU and by 
ERDF). This work was carried out in part at the Centre Esther Koplowitz, Barcelona, CERCA Programme/Generalitat de Catalunya. This work was funded by a "Proyecto de Investigación en Salud” (PI15/00587 to SL, and AS; PI15/00061 to PV; PI18/01030 to SL and AS; PI14/01126 and PI17/01019 to JF; and JR16/00006; MV17/00021; PI17/01228; RD16/0015/0003 to E.H.M.-L), integrated into the Plan Estatal de Investigación Científica y Técnica de Innovación I+D+I and co-funded by the Instituto de Salud Carlos III, subdirección General de Evaluación and the Fondo Europeo de Desarrollo Regional (FEDER, "Otra manera de hacer Europa"), by the Red Española de Esclerosis Múltiple (REEM - RD16/0015/0002, RD16/0015/0003, RD12/0032/0002, RD12/0060/01-02), by TEVA Spain, the Ayudas Merck de Investigación 2017 from the Fundación Merck Salud and the Proyecto Societat Catalana Neurologia 2017. By the MS Innovation GMSI, 2016 to E.H.M.-L. This work was also supported by the CIBERNED program (Program 1, Alzheimer Disease and SIGNAL study, www.signalstudy.es) and the National Institutes of Health (NIA grants 1R01AG056850-01A1; R21AG056974 and R01AG061566 to JF), Fundació La Marató de TV3 (20142030 to E.H.M.-L and 20141210 to JF); in addition, the Fundació Catalana Síndrome de Down and Fundació Víctor Grífols i Lucas partially supported this work. This work was also supported by the Generalitat de Catalunya (SLT006/17/00119 to JF). E.L.-S. holds a pre-doctoral grant from the University of Barcelona (APIF). C.M. was awarded by the Hospital Clinic Emili Letang and she holds a P-FIS contract (FI19/00111). J.R. holds a Miguel Servet Research Contract (CPII19/00009) and Research Project PI19/00394 from the Plan Nacional de I+D+I 2013-2016, the Instituto de Salud Carlos III-Subdirección General de Evaluación y Fomento de la Investigación and the European Regional Development Fund (FEDER, 'Investing in your future'). The funding bodies had no role in the design and performance of the study; the collection, management, analysis and interpretation of the data; the preparation, revision or approval of the manuscript; and the decision to submit the manuscript for publication.

\section{Author contribution}

E.S., E.M.-H. and S.L. conceived the experiments. V.M., E.V., M.A., J.R., J.F., F.P. and J.B. provided methodological support. E.S., E.M.-H. and V.M. carried out the experiments. M.S., N.S.-V., C.M., Y.B., I.P.-V., A.S., E.H.M.-L, P.V. and S.L. recruited the subjects and performed the clinical assessments. E.S., V.M., E.V. carried out the statistical analysis. E.S., E.M.-H., E.L.-S. drafted the manuscript, which all the authors reviewed and approved.

\section{Competing interests Statement}

E.M.-H., V.M., E.V., J.R., C.M., J.B. and F.P. have nothing to disclose. E.S and E.L.-S received travel reimbursement from Sanofi; N.S.-V. received compensation for consulting services and speaker honoraria from GenzymeSanofi, Novartis, Roche and Almirall; Y.B. received speaking honoraria from Biogen, Novartis and Genzyme; I.P.-V. received travel reimbursement from Roche and Genzyme, and she holds stock options in Aura Innovative Robotics. Currently, she is an employee at UCB Pharma, her contribution to this work is associated with her previous work at IDIBAPS; M.S. received speaker honoraria from Genzyme, Novartis and Biogen; M.A. holds equity shares of Bionure, S.L. and Goodgut S.L. and stock options of Attune Neurosciences Inc. He is currently an employee of Roche, although his contribution to this work is associated with his previous work at IDIBAPS; E.H.M.-L. received travel support for international and national meetings from Roche and Sanofi-Genzyme, and honoraria for consultancies from Novartis, Roche and Sanofi. She is currently employed by the European Medicines Agency (Human Medicines) since 16 April 2019. This article is related with her activity under Hospital Clinic of Barcelona/IDIBAPS affiliation and consequently, as external activity, it does not represent the views of the Agency or its Committees. She is a member of the International Multiple Sclerosis Visual System 
(IMSVISUAL) Consortium; A.S. received compensation for consulting services and speaker honoraria from Bayer-Schering, Merck-Serono, Biogen-Idec, Sanofi-Aventis, TEVA, Novartis and Roche; J.F. has received compensation for consultancies to Novartis, Lündbeck and AC Immune; S.L. received compensation for consulting services and speaker honoraria from Biogen Idec, Novartis, TEVA, Genzyme, Sanofi and Merck.

\section{References}

1. Rocca, M. A. et al. Clinical and imaging assessment of cognitive dysfunction in multiple sclerosis.Lancet Neurol.14,302-317(2015).

2. Mangeat, G. et al. Changes in structural network are associated with cortical demyelination in early multiple sclerosis.Hum. Brain Mapp.39,2133-2146(2018).

3. Calabrese, M. et al. Exploring the origins of grey matter damage in multiple sclerosis.Nat. Rev. Neurosci.16,147-158(2015).

4. Lassmann, H. Multiple Sclerosis Pathology.Cold Spring Harb. Perspect. Med.8,(2018).

5. Yaldizli, Ã. et al. Characteristics of lesional and extra-lesional cortical grey matter in relapsing-remitting and secondary progressive multiple sclerosis: A magnetisation transfer and diffusion tensor imaging study.Mult. Scler.22,150-159(2016).

6. Llufriu, S. et al. Cognitive functions in multiple sclerosis: impact of gray matter integrity.Mult. Scler.20,424432(2014).

7. Eshaghi, A. et al. Progression of regional grey matter atrophy in multiple sclerosis.Brain141,16651677(2018).

8. Bester, M. et al. Non-Gaussian diffusion MRI of gray matter is associated with cognitive impairment in multiple sclerosis.Mult. Scler.21,935-944(2015).

9. Jonkman, L. E., Klaver, R., Fleysher, L., Inglese, M. \& Geurts, J. J. The substrate of increased cortical FA in MS: A 7T post-mortem MRI and histopathology study.Mult. Scler.22,1804-1811(2016).

10. Andorra, M. et al. Assessing Biological and Methodological Aspects of Brain Volume Loss in Multiple Sclerosis.JAMA Neurol.75,1246-1255(2018).

11. Tsagkas, C. et al. Longitudinal patterns of cortical thinning in multiple sclerosis.Hum. Brain Mapp.41,21982215(2020).

12. Montal, V. et al. Cortical microstructural changes along the Alzheimer's disease continuum.Alzheimers. Dement.14,340-351(2018).

13. Illán-Gala, I. et al. Cortical microstructure in the behavioural variant of frontotemporal dementia: looking beyond atrophy.Brain142,1121-1133(2019).

14. Montal, V. et al. Biphasic cortical macro- and microstructural changes in autosomal dominant Alzheimer's disease.Alzheimers. Dement.(2020)doi:10.1002/alz.12224.

15. Stock, B. et al. Distribution of Cortical Diffusion Tensor Imaging Changes in Multiple Sclerosis.Frontiers in Physiologyvol.11(2020).

16. Granberg, T. et al. In vivo characterization of cortical and white matter neuroaxonal pathology in early multiple sclerosis.Brain140,2912-2926(2017). 
17. Schmierer, K. et al. Diffusion tensor imaging of post mortem multiple sclerosis brain.Neuroimage35,467477(2007).

18. Lucchinetti, C. F. et al. Inflammatory cortical demyelination in early multiple sclerosis.N. Engl. J. Med.365,2188-2197(2011).

19. Eshaghi, A. et al. Deep gray matter volume loss drives disability worsening in multiple sclerosis.Ann. Neurol.83,210-222(2018).

20. Haider, L. et al. Multiple sclerosis deep grey matter: the relation between demyelination, neurodegeneration, inflammation and iron.J. Neurol. Neurosurg. Psychiatry85,1386-1395(2014).

21. Kipp, M. et al. Thalamus pathology in multiple sclerosis: from biology to clinical application.Cell. Mol. Life Sci.72,1127-1147(2015).

22. Klaver, R. et al. Neuronal and axonal loss in normal-appearing gray matter and subpial lesions in multiple sclerosis.J. Neuropathol. Exp. Neurol.74,453-458(2015).

23. Llufriu, S. et al. Structural networks involved in attention and executive functions in multiple sclerosis.Neuroimage Clin13,288-296(2017).

24. Polman, C. H. et al. Diagnostic criteria for multiple sclerosis: 2010 revisions to the McDonald criteria.Ann. Neurol.69,292-302(2011).

25. Scalfari, A., Neuhaus, A., Daumer, M., Muraro, P. A. \& Ebers, G. C. Onset of secondary progressive phase and long-term evolution of multiple sclerosis.J. Neurol. Neurosurg. Psychiatry85,67-75(2014).

26. Rao, S. M., Leo, G. J., Bernardin, L. \& Unverzagt, F. Cognitive dysfunction in multiple sclerosis. I. Frequency, patterns, and prediction.Neurology41,685-691(1991).

27. Sepulcre, J. et al. Cognitive impairment in patients with multiple sclerosis using the Brief Repeatable Battery-Neuropsychology test.Mult. Scler.12,187-195(2006).

28. Martínez-Heras, E. et al. Improved Framework for Tractography Reconstruction of the Optic Radiation.PLoS One10,e0137064(2015).

29. Prados, F. et al. A multi-time-point modality-agnostic patch-based method for lesion filling in multiple sclerosis.Neuroimage139,376-384(2016).

30. Klein, A. \& Tourville, J. 101 Labeled Brain Images and a Consistent Human Cortical Labeling Protocol.Frontiers in Neurosciencevol.6(2012).

31. Patenaude, B., Smith, S. M., Kennedy, D. N. \& Jenkinson, M. A Bayesian model of shape and appearance for subcortical brain segmentation.Neuroimage56,907-922(2011).

32. Smith, S. M. et al. Advances in functional and structural MR image analysis and implementation as FSL.Neuroimage23 Suppl 1,S208-19(2004).

33. Tournier, J. D. et al. MRtrix3: A fast, flexible and open software framework for medical image processing and visualisation.Neuroimage116137(2019).

34. Basser, P. J., Mattiello, J. \& LeBihan, D. MR diffusion tensor spectroscopy and imaging.Biophys. J.66,259267(1994).

35. Illán-Gala, I. et al. Cortical microstructure in the amyotrophic lateral sclerosis-frontotemporal dementia continuum.Neurology95,e2565-e2576(2020). 
36. Greve, D. N. \& Fischl, B. Accurate and robust brain image alignment using boundary-based registration.Neuroimage48,63-72(2009).

37. Fortin, J. P. et al. Harmonization of multi-site diffusion tensor imaging data.Neuroimage161,149170(2017).

38. Radua, J. et al. Increased power by harmonizing structural MRI site differences with the ComBat batch adjustment method in ENIGMA.Neuroimage116956(2020).

39. Marinescu, R. V., Eshaghi, A., Alexander, D. C. \& Golland, P. Asoftwareforthevisualisationofbrainstructures,biomarkersandassociatedpathologicalprocesses. Multimodal Brain Image Anal Math Found Comput Anat (2019)11846,112-120(2019).

\section{Tables}

Table 1. Demographic, clinical, and cognitive data of the study population.

\begin{tabular}{|c|c|c|c|c|c|}
\hline & \multirow{2}{*}{$\begin{array}{l}\text { Healthy } \\
\text { controls } \\
(n=54)\end{array}$} & \multicolumn{4}{|c|}{ Multiple sclerosis patients } \\
\hline & & $\begin{array}{l}\text { MS1 } \\
(n=67)\end{array}$ & $\begin{array}{l}\text { MS2 } \\
(n=107)\end{array}$ & $\begin{array}{l}\text { MS } 3 \\
(n=73)\end{array}$ & $p$ value \\
\hline \multicolumn{6}{|l|}{ Demographic data } \\
\hline Female, n (\%) & $35(65)$ & $49(73)$ & $75(70)$ & $48(66)$ & $0.709^{a}$ \\
\hline Age (years) & $38.5(10.3)$ & $36.8(8.9)$ & $40.9(8.8)$ & $50.2(8.0)$ & $<0.001^{b}$ \\
\hline \multicolumn{6}{|l|}{ Clinical data } \\
\hline \multicolumn{6}{|l|}{ MS type, n (\%) } \\
\hline Relapsing-remitting & - & $67(100)$ & $102(95)$ & $58(79)$ & \multirow[t]{2}{*}{$<0.001^{\mathrm{a}}$} \\
\hline Secondary progressive & - & $0(0)$ & $5(5)$ & $15(21)$ & \\
\hline Disease duration, median (IQR) & - & $1.1(0.2-2.9)$ & $\begin{array}{l}10.2(7.4- \\
12.3)\end{array}$ & $\begin{array}{l}21.2(17.2- \\
27.0)\end{array}$ & $<0.001^{\mathrm{c}}$ \\
\hline $\begin{array}{l}\text { Number of relapses, median } \\
\text { (IQR) }\end{array}$ & - & $2(1-2)$ & $3(3-5)$ & $5(3-9)$ & $<0.001^{\mathrm{c}}$ \\
\hline \multirow[t]{2}{*}{ EDSS score, median (range) } & \multirow[t]{2}{*}{-} & 1.5 & 1.5 & 2.5 & \multirow[t]{2}{*}{$<0.001^{\mathrm{a}}$} \\
\hline & & $(0-5.5)$ & $(0-6.5)$ & $(1.0-7.0)$ & \\
\hline Current use of DMT, n (\%) & - & $12(18)$ & $69(67)$ & $39(58)$ & $<0.001^{a}$ \\
\hline $\begin{array}{l}\text { zAttention-Processing speed, } \\
\text { median (IQR) }\end{array}$ & - & $\begin{array}{l}-0.092 \\
(-0.88-0.5)\end{array}$ & $\begin{array}{l}0.257(-0.33- \\
0.57)\end{array}$ & $\begin{array}{l}0.087(-0.75- \\
0.68)\end{array}$ & $0.117^{c}$ \\
\hline
\end{tabular}

Continuous variables are given as the mean (SD). DMT, Disease Modifying Treatment; EDSS, Expanded Disability Status Scale; IQR, Interquartile range; MS, multiple sclerosis.

${ }^{a}$ Chi-squared test; ${ }^{b}$ one-way Anova test; ${ }^{c}$ Kruskal-Wallis test. 


\section{Figures}

STRUCTURAL MRI PROCESSING

a) MS lesion filling
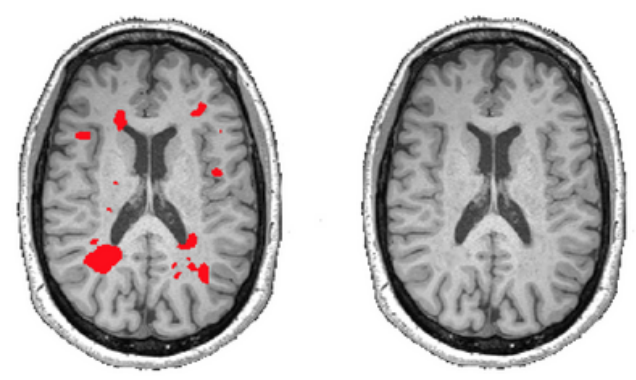

b) Corticall parcellation on the pial surface

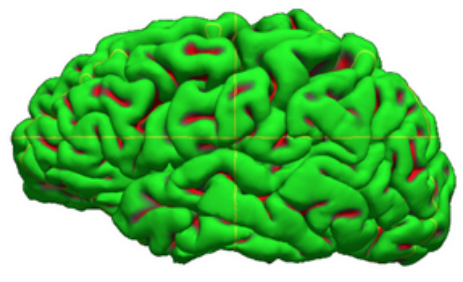

c) Cortical and subcortical parcellations

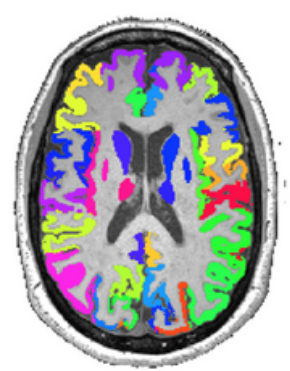

\section{DIFFUSION MRI PROCESSING}

d) DWI-preprocessing
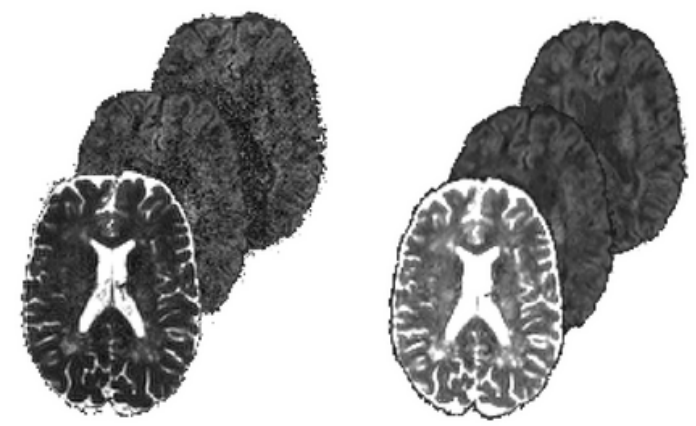

e) MD diffusion tensor map

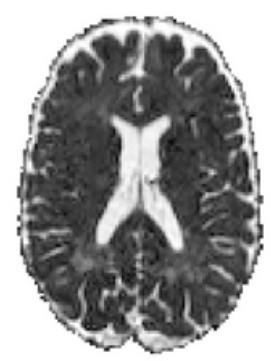

f) Quantification of MD in GM parcellation
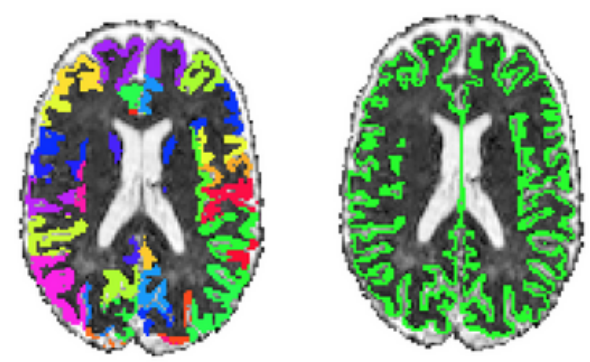

\section{Figure 1}

Flowchart for the structural (left) and diffusion MRI processing (right). To quantify GM volume (left), WM lesions in the 3D-MPRAGE were segmented using 3D-FLAIR as a reference and a WM lesion-filling approach was applied (a). We parcellated both the cortical pial surface (b), and the cortical and subcortical GM regions, to obtain volumetric values in 76 regions. To calculate the diffusion metrics in each region (right), after performing the standard diffusion pre-processing steps (d) we obtained the mean diffusivity metric (e), which was realigned to the cortical and subcortical surface on the diffusion tensor imaging space (f). 
Right hemisphere

a) MD
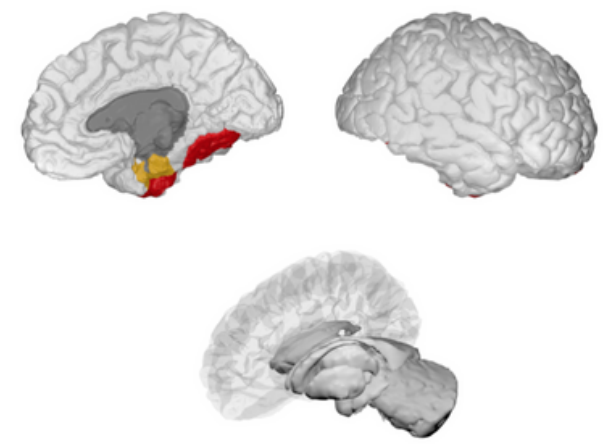

b) Volume
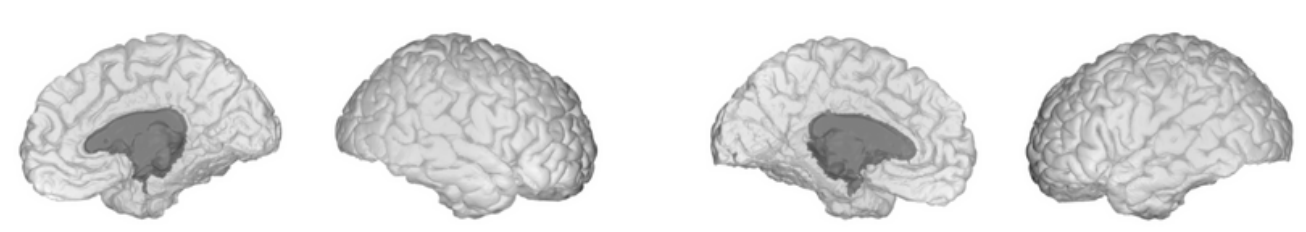

Very low
$(<0.2)$

Left hemisphere
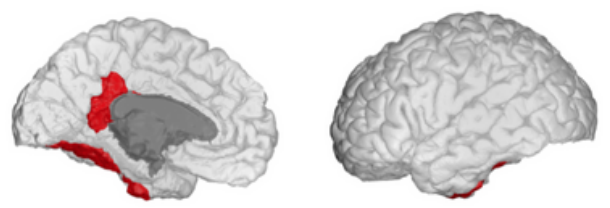

Large

$(>0.8)$

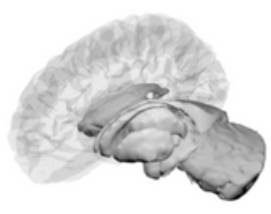

Medium

(0.5 -0.8)

Low

(0.2-0.5)
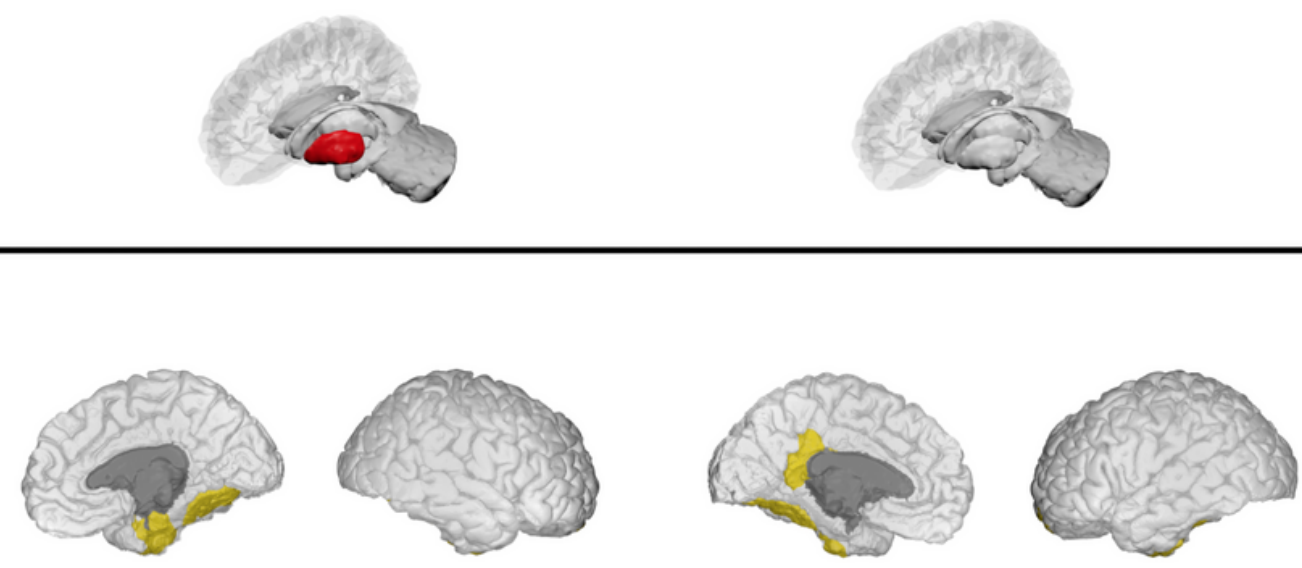

MD
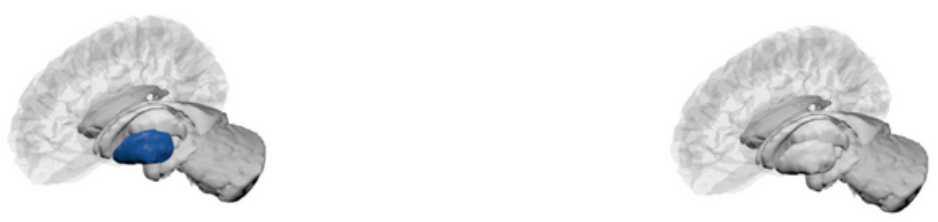

Volume

Overlap

\section{Figure 2}

Modifications in patients with less than 5 years of disease duration. Differences in the grey matter mean diffusivity (a) and in volume (b) between patients from the MS1 group and HC. The colour gradient from yellow to red indicates the effect size ranges. The bottom figure shows the changes in MD (in yellow), volume (in blue) and their overlap (in green). The figure was generated using BrainPainter software.39 
Right hemisphere

a) MD
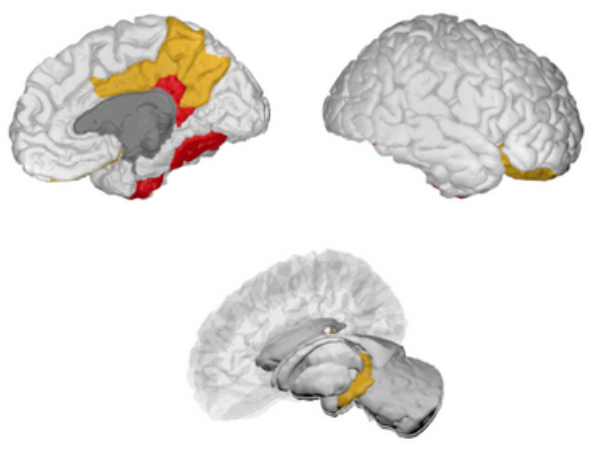

b) Volume
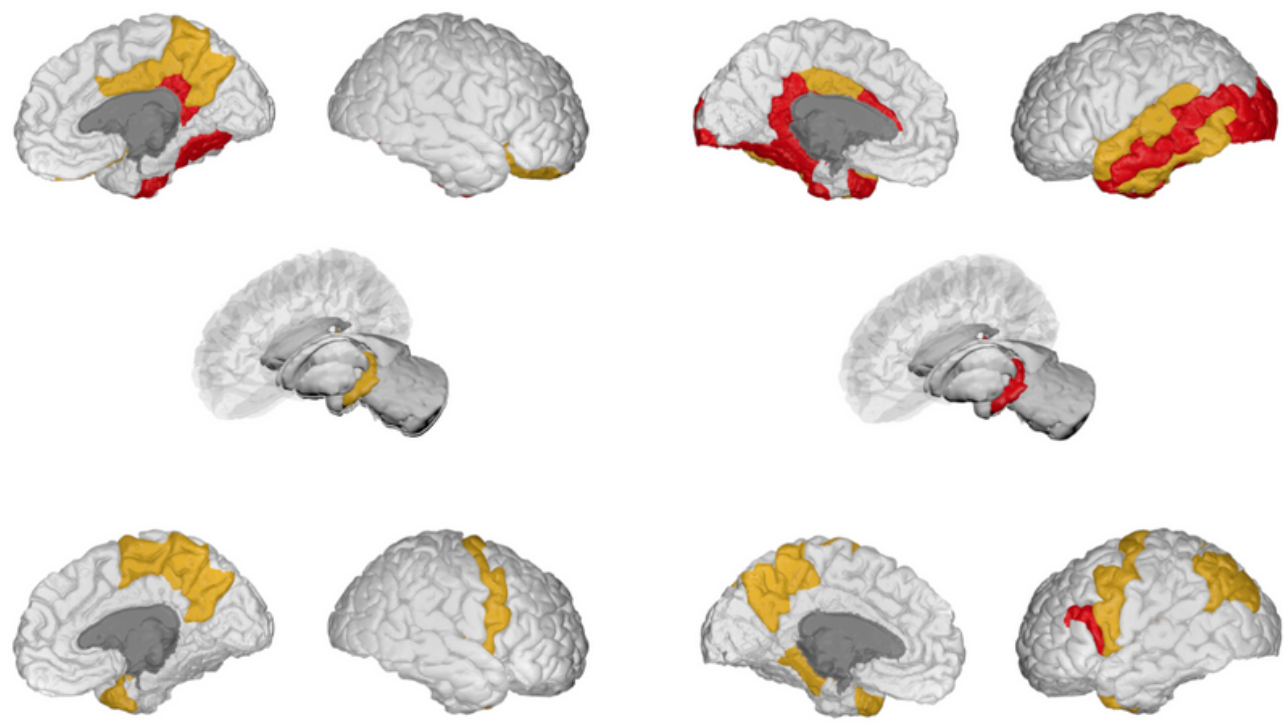

$(>0.8)$

Medium

$(0.5-0.8)$

Low

(0.2-0.5)

Very low $(<0.2)$
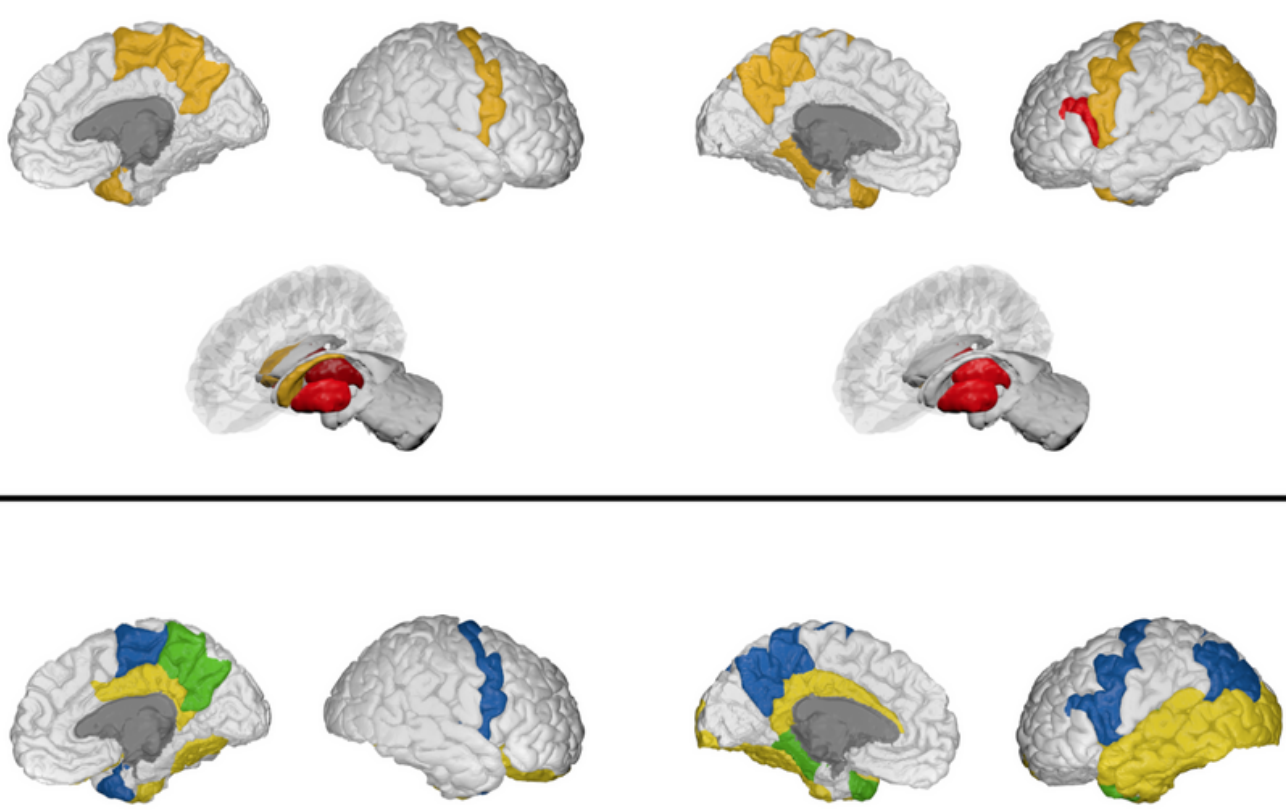

MD
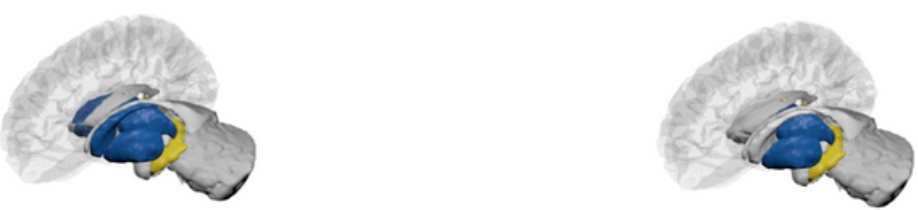

Volume

Overlap

Figure 3

Modifications in patients with 5 to 15 years of disease duration. Differences in the grey matter mean diffusivity (a) and in volume (b) between MS patients from the MS2 group and HC. The colour gradient from yellow to red indicates the effect size ranges. The bottom figure shows the changes in MD (in yellow), volume (in blue) and their overlap (in green). The figure was generated using BrainPainter software.39 
Right hemisphere

a) $M D$
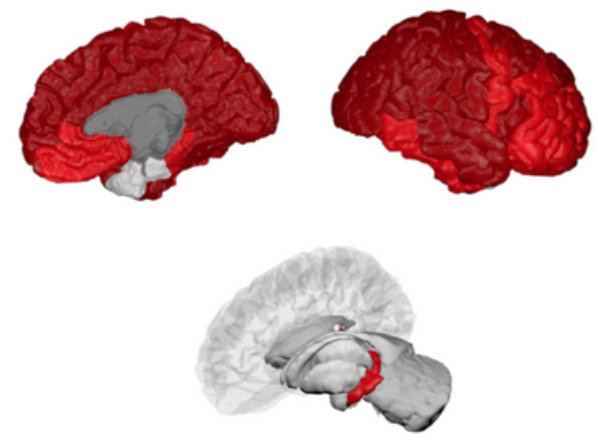

b) Volume
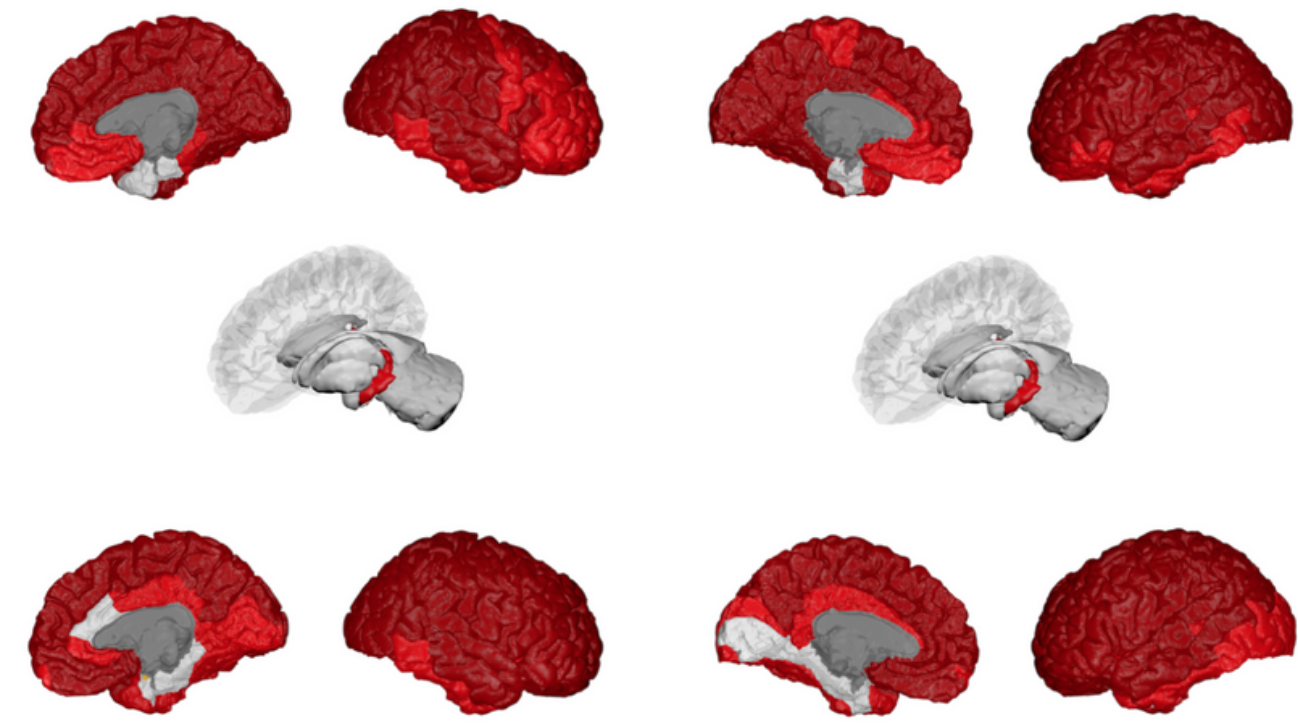

Large

$(>0.8)$

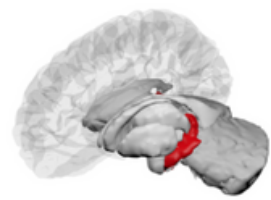

Medium

(0.5-0.8)

Low

$(0.2-0.5)$

Very low $(<0.2)$
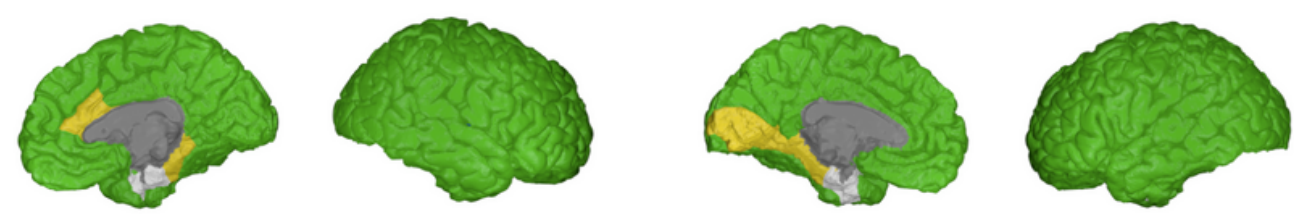

MD
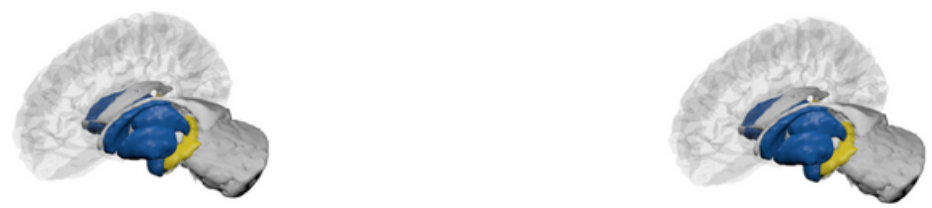

Volume

Overlap

\section{Figure 4}

Modifications in patients with more than 15 years of disease duration. Differences in the grey matter mean diffusivity (a) and in volume (b) between MS patients from the MS3 group and HC. The colour gradient from yellow to red indicates the effect size ranges. The bottom figure shows the changes in MD (in yellow), volume (in blue) and their overlap (in green). The figure was generated using BrainPainter software.39

\section{Supplementary Files}

This is a list of supplementary files associated with this preprint. Click to download.

- SolanaMartinezHerasMontalSuppMaterial.docx 